\title{
Olfactory neuroepithelium in the superior and middle turbinates: which is the optimal biopsy site?
}

\author{
Fabio de Rezende Pinna', Bruno Ctenas², Raimar Weber ${ }^{3}$, Paulo Hilario Saldiva ${ }^{4}$, Richard Louis Voegels 5. \\ 1) $\mathrm{PhD}$. Faculty Member of Department of Otorhinolaryngology, Clinics Hospital, School of Medicine, University of São Paulo, São Paulo, Brazil. \\ 2) MD (medical doctor). Department of Pathology, School of Medicine, University of São Paulo, São Paulo, Brazil. \\ 3) $\mathrm{PhD}$. Department of Otorhinolaryngology, Clinics Hospital, School of Medicine, University of São Paulo, São Paulo, Brazil. \\ 4) $\mathrm{PhD}$. Associate Professor in the Department of Pathology, School of Medicine, University of São Paulo, São Paulo, Brazil \\ 5) PhD. Associate Professor in the Department of Otorhinolaryngology, Clinics Hospital, School of Medicine, University of São Paulo, São Paulo, Brazil. \\ Fabio de Rezende Pinna \\ Department of Otorhinolaryngology, Clinics Hospital, School of Medicine, University of São Paulo, São Paulo, Brazil. Fabio de Rezende Pinna, Rua Tenente Negrão, $140 /$ \\ 91, São Paulo, SP 04530-000, Brazil. Phone +55 1181069456. \\ Fabio Pinna's work was supported by CNPq, grant number 140958/2005-3, the National Council for Scientific and Technological Development. \\ Article received on September 2, 2012. Article accepted on December 3, 2012.
}

\section{SUMMARY}

Introduction: Olfactory neuroepithelium (ON) biopsy has several therapeutic applications for both disorders of olfaction and neurodegenerative diseases. Successful collection of $\mathrm{ON}$ is still anything but routine due to a dearth of studies on the distribution of $\mathrm{ON}$ in the superior and middle turbinates.

Aim: To determine the location in which $\mathrm{ON}$ is most likely to be present in endoscopically removed cadaver superior and middle turbinates as well as the influences of gender, age, and naris side on the presence of ON and the extent to which it is present. Methods: We conducted a prospective anatomical study. The superior and middle turbinates on both sides endoscopically removed from 25 fresh cadavers (less than $12 \mathrm{~h}$ post-mortem). The turbinates were halved into anterior and posterior segments for a total of 200 specimens, which were analyzed after hematoxylin and eosin and immunohistochemical staining. Hematoxylin and eosin-stained slides were subjected to blind examination by 3 independent pathologists, and the presence of ON was graded on a 5-point scale from 0 to 4 . Kappa measurement was used to determine the agreement between pairs of observers.

Results: ON was present in $82.9 \%$ of superior turbinate samples and in $17.1 \%$ of middle turbinate samples. Immunohistochemistry detected ON in superior turbinates only by S-100 staining and only in 15 fragments. Gender, age, and naris side had no statistically significant effects on the presence of ON.

Conclusion: When biopsying ON, the posterior portion of the superior turbinate should be targeted whenever possible because it has the highest concentration of ON among the nasal structures.

Keywords: Olfactory Mucosa; Olfaction Disorders; Turbinates; Biopsy; Hematoxylin; Immunohistochemistry.

\section{INTRODUCTION}

Olfactory disturbances significantly impair the patient's quality of life (1-4). The perception of smell occurs when odorant particles are inhaled and bind to olfactory receptors located in the olfactory neuroepithelium (ON) (5). The nerve endings in this tissue have a unique capacity to regenerate $(5,6)$, which makes ON a prime target for studies seeking to understand the pathophysiology of olfactory disturbances (4, 6-10) and several neurological disorders, such as Alzheimer's disease, spinal trauma, and a variety of storage diseases (11-14). ON is found in the olfactory area that comprises the cribriform plate, the medial portion of the superior turbinate (ST), and the superior portion of the nasal septum $(3,5)$; it may also be found on the medial surface of the middle turbinate (MT) (3-5, 15-17). Biopsies of the olfactory mucosa $(6,11,13)$, particularly those obtained endoscopically (10, 18), constitute a source of true neural progenitor cells with the added bonus of extracranial location (18). Although biopsy of the olfactory mucosa is an established (if experimental) procedure, successful in vivo collection of $\mathrm{ON}$ is anything but routine. This may be attributed in part to the lack of post-mortem studies of ON distribution under endoscopic visualization. Few studies have described the presence of this tissue with respect to age, gender, and symmetry ( 8 , $16,19)$, and none has focused on its distribution on the medial surfaces of the STs and MTs. Although Say (20) has reported that removal of the inferior third of the ST has no effect on olfaction, there have been no studies published on which region has the highest concentration of ON and, therefore, there is no information on which region is most likely to suffer injury leading to ON damage during endoscopic surgery on the nasal cavity.

The objective of this study was to conduct a detailed assessment of the distribution of ON on the STs and MTs 
of fresh cadavers through hematoxylin and eosin (H\&E) staining and immunohistochemistry. We sought to detect the most likely site for ON to be obtained by biopsy.

\section{METHOD}

This study was approved by the Ethics Commission of the University of São Paulo School of Medicine Clinics Hospital. We dissected 25 fresh cadavers (2-12 h postmortem) ranging in age from 30 to 85 years. The cadavers were stored in a cooling chamber below $4^{\circ} \mathrm{C}\left(39^{\circ} \mathrm{F}\right)$ until dissection. We began by performing craniotomy, removing the brain (including the olfactory bulb), inspecting the anterior fossa, and examining the full extent of the nasal cavity under endoscopy, paying particular attention to the olfactory area.

We excluded cadavers in which death had occurred more than $12 \mathrm{~h}$ before dissection and those with evidence or history of nasal trauma, fractures, or surgery, signs of nasal polyps or purulent discharge on endoscopy, or a history of neurodegenerative disease, chemotherapy or radiotherapy, or inflammatory or neoplastic disease of the olfactory bulb.

The superior turbinates were endoscopically displaced at their posterior insertion onto the anterior sphenoid wall, cut at their superior insertion onto the base of the skull, and removed bilaterally. We attempted to minimize manipulation of the medial surfaces of the STs during dissection (Figure 1). After ethmoidectomy, the entire vertical insertion of the MT onto the base of the skull was also removed on both sides. Again, maximal preservation of the medial surfaces was attempted.

After resection, the anteroposterior length of each turbinate was measured with a ruler, and the turbinates were then sliced in half. All mucosa was stripped from the medial surface of each half of each ST and MT specimen. We obtained 8 mucosal fragments from different anatomical locations from each of the 25 cadavers for a total of 200 fragments. Two of these fragments, both from the anterior ST, could not be examined due to complete autolysis of their epithelium (Table 1).

The specimens were fixed in 10\% formaldehyde solution and embedded in paraffin. Blocks were then sectioned crosswise into 3 - $\mu \mathrm{m}$ slices, which were stained with H\&E.

For immunohistochemistry, 3- $\mu$ m sections were cut and mounted on 3-aminopropyltriethoxysilane (APTES)treated slides. We used anti-neuron-specific beta-III tubulin,

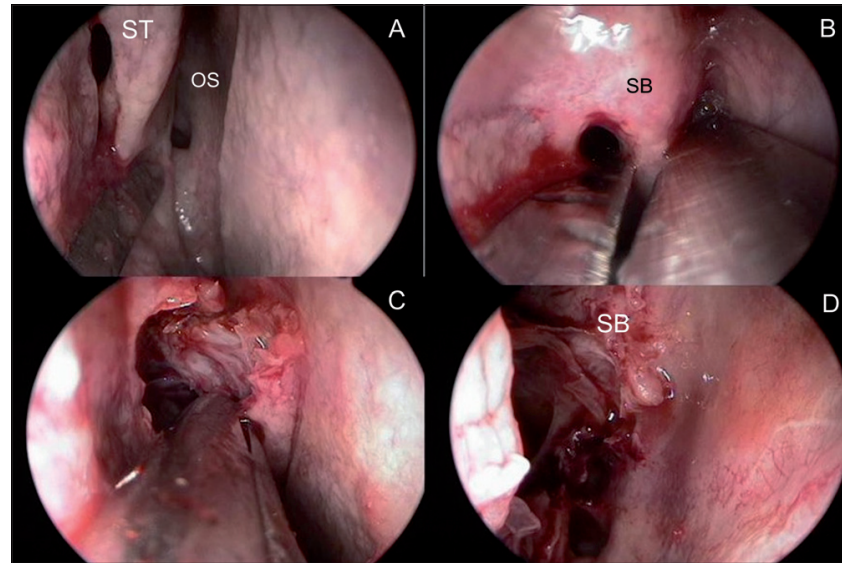

Figures 1A, 1B, 1C, 1D. 1A: Identification of the right superior turbinate through identification of the natural ostium; 1B: Dissection of the insertion of the right superior turbinate onto the base of the skull; 1C: Removal of the superior turbinate by its lateral surface; 1D: Final aspect of the base of the skull after removal of the superior turbinate. ST, superior turbinate; OS, sphenoid sinus natural ostium; SB, skull base.

Table I. Numerical codes for the identification of each analyzed fragment.

\begin{tabular}{ccccc}
\hline Side & Turbinate & Half & $\begin{array}{c}\text { Numerical } \\
\text { code }\end{array}$ & $\begin{array}{c}\text { No. offragments } \\
\text { analyzedhistologically }\end{array}$ \\
\hline Left & Superior & Posterior & 1 & 25 \\
& & Anterior & 2 & $23 *$ \\
\multirow{4}{*}{ Middle } & Posterior & 3 & 25 \\
& & Anterior & 4 & 25 \\
\multirow{4}{*}{ Superior } & Posterior & 5 & 25 \\
& \multirow{2}{*}{ Middle } & Anterior & 6 & 25 \\
& Posterior & 7 & 25 \\
& Anterior & 8 & 25 \\
\hline
\end{tabular}

*Two fragments of anterior left superior turbinate were unsuitable for analysis.

anti-microtubule-associated protein 5, anti-neu-n, antichromogranin, anti-synaptophysin, and anti-S-100 protein antibodies; positive labeling was detected only for the last. Due to the inherent difficulties of using immunohistochemical methods on cadaver tissue, we were only able to label 15 fragments successfully. To distinguish ON from respiratory epithelium in H\&E-stained specimens, we used the same criteria employed by Kern in 2000 (23): presence of nerve fascicles in the lamina propria, presence of irregular cilia, presence of a cellular lamina propria with few vascular structures, presence of pseudostratified epithelium, absence of goblet cells, absence of a thick basal membrane, and positive immunohistochemical labeling (Figure 2). Meeting 4 of these 7 criteria was sufficient to confirm the presence of $\mathrm{ON}$ in a specimen. 


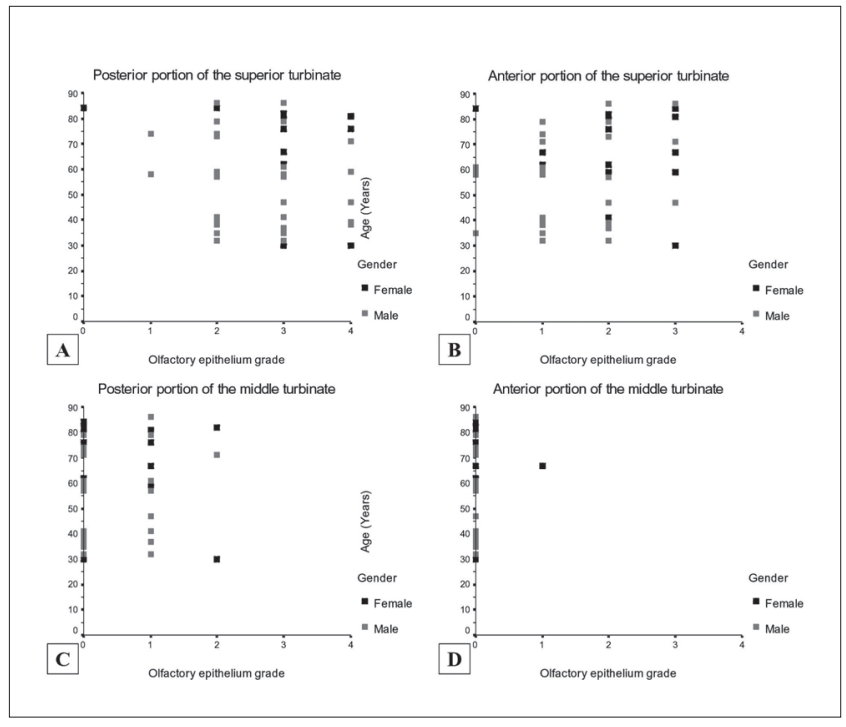

Figures 2A, 2B, 2C, 2D. 2A: Light micrograph (400x magnification) of a grade 0 anterior middle turbinate sample. This slide contains only respiratory epithelium; note the predominance of goblet cells. 2B: Light micrograph of a posterior superior turbinate fragment with a predominance of olfactory neuroepithelium. Note the isolated focus of respiratory epithelium between the arrows. This specimen was graded as 3+. 2C: Light micrograph of a posterior superior turbinate fragment, lower magnification. Olfactory neuroepithelium predominates in this specimen; note the thin basal membrane and cellular lamina propria. 2D: Light micrograph of a posterior middle turbinate fragment, $200 \mathrm{x}$ magnification. This specimen contains mostly respiratory epithelium. Note the thick basal membrane and predominance of vascular structures in the lamina propria.

The distribution of neuroepithelium was graded on a scale of 0 to 4 according to the prevalence of $\mathrm{ON}$ in each H\&E-stained slide. This semi-quantitative method is commonly used by pathologists for analyzing tumor cellularity (24).

Each grade of the scale corresponds to a percent bracket of ON distribution, as shown in Table 2 .

In an attempt to decrease the subjectivity of our methods, blind examination of each fragment was performed by 3 independent pathologists. The mean of the grades assigned to each slide was used for data analysis.

We determined agreement between the pairs of pathologists using kappa statistics (25) (data not shown, interpretation in the Discussion section). Table 3 shows the percentages of agreement between all, 2 , or no pathologists on the ON grade assigned to each slide broken down by the anatomical region of origin.
Table 2. Grading system for the prevalence of olfactory neuroepithelium $(\mathrm{ON})$ in each fragment.

\begin{tabular}{cc}
\hline Grade & Percentage of ON infragment \\
\hline 0 & Absent \\
1 & $>0 \%$ and $\leq 25 \%$ \\
2 & $>25 \%$ and $\leq 50 \%$ \\
3 & $>50 \%$ and $\leq 75 \%$ \\
4 & $>75 \%$ and $\leq 100 \%$ \\
\hline
\end{tabular}

Table 3. Inter-rater agreement according to the anatomical origin of the analyzed fragment.

\begin{tabular}{lcccr}
\hline & & \multicolumn{3}{c}{ Pathologists agreeing } \\
Turbinate & Quadrant & All & 2 & None \\
\hline Middle & Posterior & $50(100.0 \%)$ & $0(0.0 \%)$ & $0(0.0 \%)$ \\
& Anterior & $50(100.0 \%)$ & $0(0.0 \%)$ & $0(0.0 \%)$ \\
Superior & Posterior & $35(70.0 \%)$ & $10(20.0 \%)$ & $5(10.0 \%)$ \\
& Anterior* & $45(93.8 \%)$ & $0(0.0 \%)$ & $3(6.2 \%)$ \\
\hline
\end{tabular}

* Two slides were excluded $(\mathrm{n}=48)$.

Quantitative variables were represented by their means and respective standard deviations and/or medians and interquartile ranges. The distributions of ON on the STs and MTs were analyzed by describing the percentages of ON of each grade in the studied anatomical regions. The right and left turbinates were considered jointly in this analysis: each cadaver provided 2 samples (right and left) from each region of each turbinate.

To assess the influence of variables such as gender, age, and laterality on the presence of ON, we carried out the following statistical tests for each half of each ST and MT sample: analysis of gender differences in ON prevalence using chi-square and Fisher's exact tests, analysis of the effect of age on the ON grade using Pearson's correlation, and analysis of concordance between the left and right sides using kappa statistics.

Differences were considered statistically significant when the $p$ values did not exceed $0.05(\alpha=0.05)$.

\section{RESULTS}

The mean age of the cadavers was 55.4 years with a standard deviation of 17.5 years for the males and 64.8 years with a standard deviation of 19 years for the females. ON was present to some degree in 111 (56.1\%) of the 198 slides analyzed. The prevalence rates of the various ON 
grades in the posterior and anterior fragments of the ST and MT specimens are shown in Table 4.

ON was present in $82.9 \%$ of the ST slides, a 4.9 -fold (CI 95\%: 3.3-7.4) higher prevalence than observed in the MT specimens (17.1\% of slides, $p<0.001)$. Comparison of the posterior ( 48 slides, $98 \%$ prevalence of $\mathrm{ON}$ ) and anterior (43 slides, 89.6\% prevalence) ST samples yielded a prevalence ratio of 1.1. When posterior fragments of the STs and MTs were compared, the prevalence ratio rose to 2.9.

We were only able to detect ON through immunohistochemistry in 15 ST specimens: 10 of posterior fragments (out of 50, 20\%) and 5 of anterior fragments (out of $48,7.6 \%)$. We were unable to detect $\mathrm{ON}$ immunohistochemically in MT specimens.

We then compared the results of H\&E staining to those obtained through immunohistochemistry for each anatomical structure studied. We found that 14 of the 15 fragments with positive S-100 protein labeling contained more than $50 \%$ ON by analysis of H\&E-stained sections.
The remaining immunohistochemistry-positive fragment was classified as grade 2 (26-50\% ON) by H\&E staining analysis. Immunohistochemistry did not detect $\mathrm{ON}$ in any fragments graded 0 or $1(0-25 \%$ prevalence of $\mathrm{ON})$ by H\&E analysis.

The amount of ON did not correlate significantly with age in any of the anatomical regions studied. The Pearson correlation coefficients were " 0.16 for the posterior ST ( $p=0.3), 0.09$ for the anterior ST $(p=0.5), 0.1$ for the posterior MT $(p=0.5)$, and 0.07 for the anterior MT ( $p$ $=0.6)$. There was also no significant association between gender and the presence and grade of ON.

As shown in Tables 5 and 6, the concordance between the right and left sides for each cadaver and each anatomical region was negligible to slight. For the ST samples, simple inter-rater agreement was only 38.8\%, with a kappa coefficient of 0.18 (standard error, $0.09 ; p=$ 0.02). Simple agreement was higher for the MT specimens at $69.2 \%$, with a kappa of 0.27 (SE, $0.13 ; p=0.01$ ) (Figure $3)$.

Table 4. Prevalence rates of each neuroepithelium grade by anatomical region.

\begin{tabular}{|c|c|c|c|c|}
\hline \multirow{4}{*}{$\begin{array}{l}\text { Olfactory } \\
\text { neuroepithelium }\end{array}$} & \multicolumn{4}{|c|}{ Turbinate } \\
\hline & \multicolumn{2}{|c|}{$\begin{array}{l}\text { Superior } \\
\text { Fragment }\end{array}$} & \multicolumn{2}{|c|}{$\begin{array}{c}\text { Middle } \\
\text { Fragment }\end{array}$} \\
\hline & Posterior & Anterior & Posterior & Anterior \\
\hline & $(n=50)$ & $(n=48)$ & $(n=50)$ & $(n=50)$ \\
\hline Absent & I (2.0\%) & $5(10.4 \%)$ & $32(64.0 \%)$ & 49 (98.0\%) \\
\hline Present & $49(98.0 \%)$ & $43(8.6 \%)$ & $18(36.0 \%)$ & I (2.0\%) \\
\hline Up to $25 \%$ & $2(4.0 \%)$ & $14(2.2 \%)$ & $15(30.0 \%)$ & I (2.0\%) \\
\hline $26-50 \%$ & $13(26.0 \%)$ & $20(41.6 \%)$ & $3(6.0 \%)$ & $0(0.0 \%)$ \\
\hline $51-75 \%$ & $23(46.0 \%)$ & $9(\mid 8.8 \%)$ & $0(0.0 \%)$ & $0(0.0 \%)$ \\
\hline$>76 \%$ & | | (22.0\%) & $0(0.0 \%)$ & $0(0.0 \%)$ & $0(0.0 \%)$ \\
\hline
\end{tabular}

Table 5. Concordance between specimens from the left and right superior turbinates as to olfactory neuroepithelium $(\mathrm{ON})$ grade.

\begin{tabular}{lcccccc}
\hline & \multicolumn{5}{c}{ ON Grade in Left Turbinate } \\
& & 0 & 1 & 2 & 3 & 4 \\
\hline ONGrade & 0 & $4(8.2 \%)$ & & & & \\
in Right & 1 & $2(4.1 \%)$ & & $3(6.1 \%)$ & $2(4.1 \%)$ & \\
Turbinate & 2 & $1(2.0 \%)$ & $3(6.1 \%)$ & $6(12.2 \%)$ & $5(10.2 \%)$ & $2(4.1 \%)$ \\
& 3 & $1(2.0 \%)$ & $1(2.0 \%)$ & $5(10.2 \%)$ & $7(14.3 \%)$ & $2(4.1 \%)$ \\
& 4 & & & $1(2.0 \%)$ & $2(4.1 \%)$ & $2(4.1 \%)$ \\
\hline
\end{tabular}

* ON, olfactory neuroepithelium. 
Table 6. Concordance between specimens from the left and rightmiddle turbinates as to olfactory neuroepithelium $(\mathrm{ON})$ grade.

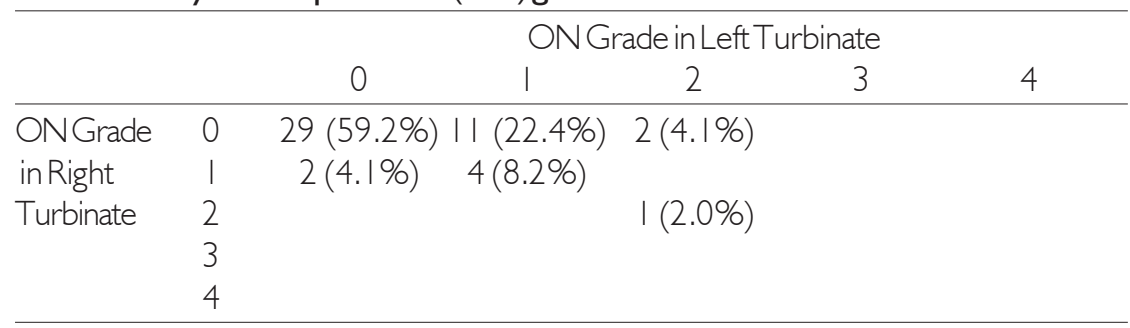

* ON, olfactory neuroepithelium.

\section{DISCUSSION}

Reliable, adequate sampling of ON during biopsy of the nasal mucosa has always presented a challenge. Biopsy of $\mathrm{ON}$ in living patients is more effective when performed endoscopically with the use of angled biopsy forceps $(6,14,26)$. Lovell reported that, in vivo, 4 to 6 samples must be taken in order to ensure detection of ON (6). As well as providing smaller samples, biopsies obtained with angled biting forceps are also subject to randomness, as the operator cannot be sure whether the biopsy site will actually contain ON $(8,27)$. ON biopsy is currently an established procedure for histopathological study of this tissue in several disorders of olfaction. Nevertheless, successful detection of $\mathrm{ON}$ is anything but routine (18).

Cadaver studies allow resection of larger portions of nasal mucosa and are therefore an interesting approach to the study of the distribution of ON $(16,19)$. According to Paik, adequate histological analysis may be accomplished up to $24 \mathrm{~h}$ post-mortem (16). We chose to conduct the present study on cadavers no more than $12 \mathrm{~h}$ post-mortem in order to decrease the risk for tissue autolysis, which commonly occurs in cadavers as a result of tissue hypoxia, and to ensure the best possible conditions for identification of ON through H\&E staining and immunohistochemistry $(10,17,28-30)$.

Nakashima and Paik reported great difficulty in determining the actual location of $\mathrm{ON}$, even in post mortem studies, most probably because they studied an extensive, poorly delimited area $(16,19)$. We therefore chose to study the distribution of ON in previously defined structures, namely the MTs andSTs. Each turbinate specimen was divided into posterior and anterior portions to give a better idea of the distribution of $\mathrm{ON}$ and of how far posteriorly surgeons should advance during biopsy. This approach also allowed us to assess the possible extent of ON damage during extended endoscopic approaches to the sella that require resection of the ST or due to

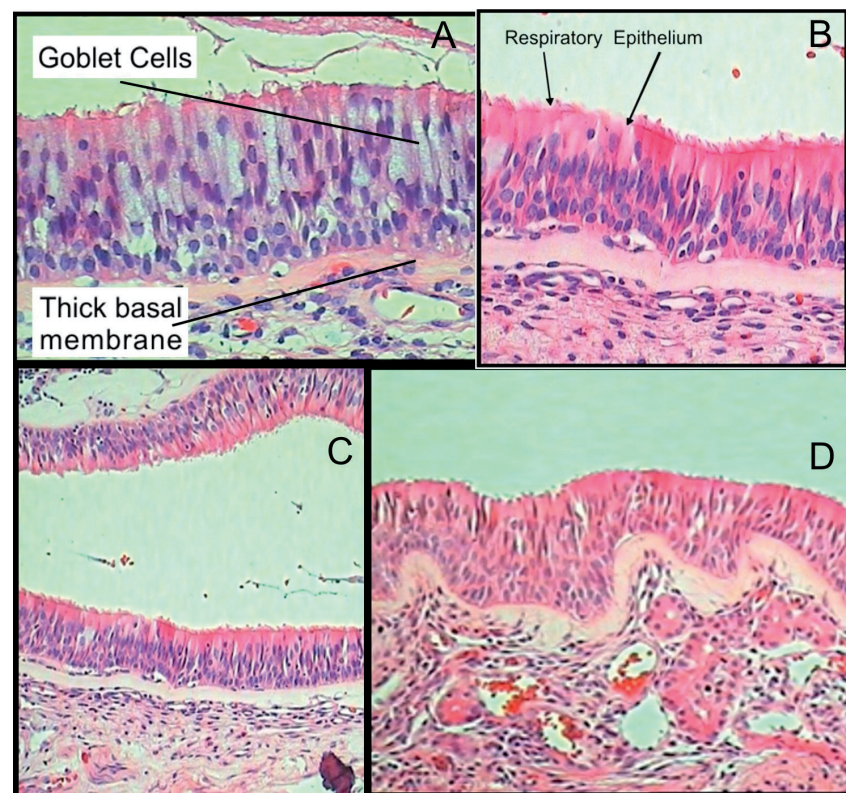

Figures 3A, 3B, 3C, 3D. Scatter plots of the correlations between the presence of $O N$ and age of the cadaver at the time of death. 6A: Posterior half of the superior turbinate; 6B: Anterior half of the superior turbinate; 6C: Posterior half of the middle turbinate; 6D: Anterior half of the middle turbinate.

inadvertent ST injury during Functional Endoscopic Sinus Surgery FESS enlargement of the sphenoid ostium.

Both H\&E staining and immunohistochemistry found the greatest amount of $\mathrm{ON}$ in the posterior ST, regardless of side. In addition to using previously described criteria to distinguish ON from respiratory epithelium in H\&E-stained sections $(23,27)$, we chose to adopt a semi-quantitative grading scheme in order to obtain more in-depth information on the distribution of $\mathrm{ON}$ in the STs and MTs. The prevalence of $\mathrm{ON}$ was graded on a 5-point scale from 0 to 4; the grading may of course have been somewhat subjective. 
We implemented 3 mechanisms to minimize the subjectivity of the results. First, the pathologists analyzed the slides blindly: the examiners did not know from which region the samples had been harvested. Second, each slide was examined by 3 independent pathologists who had no contact with each other and did not communicate results among themselves. Finally, we employed immunohistochemistry for confirmation of the H\&E results.

For the ST samples, the agreement between pathologists was $70 \%$ for the posterior and $93.8 \%$ for the anterior fragments. The inter-rater agreement was excellent (25) for anterior ST fragments and for both posterior and anterior MT fragments. For posterior ST fragments (those with the highest prevalence of $\mathrm{ON}$ ), the agreement between examining pathologists ranged from sufficient to excellent (25), confirming the reproducibility of our method for identifying ON through H\&E staining.

H\&E staining was therefore an excellent method for detecting $\mathrm{ON}$ in fragments in which its prevalence was below grade 3 , such as MT and anterior ST fragments. The inter-rater agreement on the grading of $\mathrm{H} \& \mathrm{E}$-stained samples from the posterior ST, where the prevalence of ON was higher, was lower but still sufficient to validate the results. There may have been a certain degree of confusion regarding the grading of some fragments, particularly those with a $60-80 \%$ prevalence of ON, as the ends of this relatively narrow range fall into different grades in our classification scheme.

Immunohistochemistry can be used to identify nerve endings, which might not be visualized by simple H\&E staining (8-10, 17, 23, 29, 31). The results of studies using immunohistochemical methods vary widely due to the different ways in which each author uses the antibodies. Although Margolis' work $(20,28)$ has led to extensive in vivo use of olfactory marker protein (OMP), immunohistochemical detection of $\mathrm{ON}$ in cadavers is nontrivial. Kim et al. (32) were unable to detect nerve endings in cadaver tissue using immunohistochemistry. We believe that our low detection rates were due to most of the antigens having already degenerated at the time of sample collection despite the samples' having been harvested less than $12 \mathrm{~h}$ post-mortem.

Despite these difficulties in the immunohistochemical study of cadaver tissue, we were able to label 15 specimens for S-100 protein. The immunohistochemistry results were markedly different from those obtained by H\&E staining: the former gave positive results for only $20 \%$ of posterior ST samples, a very low detection rate. S-100 protein was only detected in fragments in which H\&E staining showed a high prevalence of ON. Despite its low sensitivity in cadaver tissue, the anti-S-100 protein staining provided valuable data that confirmed that our H\&E staining-based classification closely approximated reality. Both H\&E staining and immunohistochemistry showed greater prevalence rates of $\mathrm{ON}$ in the posterior portions of ST samples.

There is a dearth of literature discussing the symmetry of the distribution of ON. Our results showed that when samples were analyzed as a whole, ON was no more prevalent in one side than in the other; however, there was no agreement between the ON distribution patterns in the right and left nares. This suggests that although the amounts of ON on each side appear to be equal, the manner in which the ON is distributed over the MTs and STs may differ between the sides. We found no correlation between decreased $\mathrm{ON}$ concentration and greater age, as has been described previously (19). Nevertheless, we must note that all of the cadavers used in our study were over the age of 30 at the time of death; had we used younger cadavers, we might have observed such a correlation.

Smoking and alcohol consumption were not used as exclusion criteria in the present study. Although previous studies have demonstrated that both habits may lead to changes in the ultrastructure of the ON, such as the loss of cilia or an increase in respiratory tissue metaplasia, the authors of these studies have emphatically noted that tobacco- and alcohol-related destruction of the $\mathrm{ON}$ is insufficiently extensive to change tissue distribution patterns because the unique regenerative capacities of ON enable it to adapt to such stimuli $(33,34)$.

\section{CONCLUSION}

In this study, ON was found to be the predominant tissue in the posterior portion of the ST, regardless of side, through both H\&E staining and immunohistochemistry. Although ON can be found on the posterior and anterior MT, its presence anywhere in the MT is exceedingly rare. Immunohistochemistry and H\&E staining may be used to complement detection of $\mathrm{ON}$ in the nasal mucosa. We suggest that clinicians approach the posterior ST when attempting biopsy of ON because ON is more likely to be found at this location. We also recommend that the posterior ST, in which ON is the most prevalent, be preserved whenever possible during endoscopic approaches to the sellar and parasellar regions.

\section{REFERENCES}

1. Schiffman SS. Taste and smell in disease (second of two parts). N Engl J Med. 1983;308:1337-43. 
2. Deems DA, Doty RL, Settle RG, et al. Smell and taste disorders, a study of 750 patients from the University of Pennsylvania Smell and Taste Center. Arch Otolaryngol Head Neck Surg. 1991;117:519-28.

3. Jones N, and Rog D. Olfaction: a review. J Laryngol Otol. 1998;112:11-24.

4. Lane AP, Gomez G, Dankulich T, et al. The superior turbinate as a source of functional human olfactory receptor neurons. Laryngoscope. 2002;112:1183-89.

5. Hadley K, Orlandi RR, and Fong KJ. Basic anatomy and physiology of olfaction and taste. Otolaryngol Clin North Am. 2004;37:1115-26.

6. Lovell MA, Jafek BW, Moran DT, et al. Biopsy of human olfactory mucosa. An instrument and a technique. Arch Otolaryngol. 1982;108:247-49.

7. Jafek BW, Murrow B, Michaels R, et al. Biopsies of human olfactory epithelium. Chem Senses. 2002;27:623-28.

8. Féron F, Perry C, McGrath JJ, et al. New techniques for biopsy and culture of human olfactory epithelial neurons. Arch Otolaryngol Head Neck Surg. 1998;124:861-66.

9. Jafek BW. Ultrastructure of human nasal mucosa. Laryngoscope. 1983;93:1576-99.

10. Lanza DC, Moran DT, Doty RL, et al. Endoscopic human olfactory biopsy technique: a preliminary report. Laryngoscope. 1993;103:815-19.

11. Ronnett GV, Leopold D, Cai X, et al. Olfactory biopsies demonstrate a defect in neuronal development in Rett's syndrome. Ann Neurol. 2003;54:206-18.

12. Ramer LM, Au E, Richter MW, et al. Peripheral olfactory ensheathing cells reduce scar and cavity formation and promote regeneration after spinal cord injury. J Comp Neurol. 2004;473:1-15.

13. Tabaton M, MonacoS, Cordone MP, et al. Prion deposition in olfactory biopsy of sporadic Creutzfeldt-Jakob disease. Ann Neurol. 2004;55:294-96.

14. Arnold SE, Lee EB, Moberg PJ, et al. Olfactory epithelium amyloid-beta and paired helical filament-tau pathology in Alzheimer disease. Ann Neurol. 2010;67(4):462-9.

15. Hart CK, Theodosopoulos PV, Zimmer LA. Olfactory changes after endoscopic pituitary tumor resection. Otolaryngol Head Neck Surg. 2010;42(1):95-7.
16. Paik SI, Lehman MN, Seiden AM, et al. Human olfactory biopsy. The influence of age and receptor distribution. Arch Otolaryngol Head Neck Surg. 1992;118:731-38.

17. Leopold DA, Hummel T, Schwob JE, et al. Anterior distribution of human olfactory epithelium. Laryngoscope. 2000;110:417-21.

18. Winstead W, Marshall CT, Lu CL, et al. Endoscopic biopsy of human olfactory epithelium as a source of progenitor cells. Am J Rhinol. 2005;19:83-90.

19. Nakashima T, Kimmelman CP, Snow JB Jr. Structure of human fetal and adult olfactory neuroepithelium. Arch Otolaryngol. 1984;110:641-46.

20. Say P, Leopold D, Cochran G, et al. Resection of the inferior superior turbinate: does it affect olfactory ability or contain olfactory neuronal tissue? Am J Rhinol. 2004;18:15760 .

21. Orlandi RR, Lanza DC, Bolger WE, et al. The forgotten turbinate: the role of the superior turbinate in endoscopic sinus surgery. Am J Rhinol. 1999;13:251-59.

22. Har-El G, Swanson RM. The superior turbinectomy approach to isolated sphenoid sinus disease and to the sella turcica. Am J Rhinol. 2001;15:149-56.

23. Kern RC. Chronic sinusitis and anosmia: pathologic changes in the olfactory mucosa. Laryngoscope. 2000;110:1071-77.

24. Layfield LJ, and Cramer H. Fine-needle aspiration cytology of intraductal papillary-mucinous tumors: a retrospective analysis. Diagn Cytopathol. 2005;32:16-20.

25. Landis JR, and Koch GG. The measurement of observer agreement for categorical data. Biometrics. 1977;33:15974.

26. Moran DT, Jafek BW, Rowley JC III, et al. Electron microscopy of olfactory epithelia in two patients with anosmia. Arch Otolaryngol. 1985;111:122-26.

27. Lee SH, Lim HH, Lee HM, et al. Olfactory mucosal findings in patients with persistent anosmia after endoscopic sinus surgery. Ann Otol Rhinol Laryngol. 2000;109:720-25.

28. Monti-Graziadei GA, Margolis FL, Harding JW, et al. Immunocytochemistry of the olfactory marker protein. J Histochem Cytochem. 1977;25:1311-6.

29. Yamagishi M, Fujiwara M, and Nakamura H. Olfactory mucosal findings and clinical course in patients with olfactory 
disorders following upper respiratory viral infection. Rhinology. 1994;32:113-8.

30. Slotnick B, Bodyak N, Davis BJ. Olfactory marker protein immunohistochemistry and the anterograde transport of horseradish peroxidase as indices of damage to the olfactory epithelium. Chem Senses. 2001;26:605-10.

31. Holbrook EH, Leopold DA, and SchwobJE. Abnormalities of axon growth in human olfactory mucosa. Laryngoscope. 2005; 115:2144-54.
32. Kim KS, Choi YS, Kim HJ, et al. The risk of olfactory disturbance from conchal plate injury during ethmoidectomy. Am J Rhinol. 2003;17:307-10.

33. Vent J, Bartels S, Haynatzki G, et al. The impact of ethanol and tobacco smoke on intranasal epithelium in the rat. Am J Rhinol. 2003;17:241-7.

34. Vent J, Robinson AM, Gentry-Nielsen MJ, et al. Pathology of the olfactory epithelium: smoking and ethanol exposure. Laryngoscope. 2004;114:1383-8. 